\title{
Comparative pathogenicity of Coxsackievirus A16 circulating and noncirculating strains in vitro and in a neonatal mouse model
}

\author{
L. Huang ${ }^{1,3 *}$, X. Liu ${ }^{1 *}$, J.L. Li ${ }^{1}$, J.L. Chang ${ }^{1}$, G.C. Liu ${ }^{1}$, X.F. Yu ${ }^{1,2}$ and W.Y. Zhang ${ }^{1}$ \\ ${ }^{1}$ Institute of Virology and AIDS Research, The First Hospital of Jilin University, Changchun, China \\ ${ }^{2}$ Department of Molecular Microbiology and Immunology, Johns Hopkins Bloomberg School of Public Health, Baltimore, MD, USA \\ ${ }^{3}$ The 208th Hospital of PLA, Changchun, China
}

\begin{abstract}
An enterovirus 71 (EV71) vaccine for the prevention of hand, foot, and mouth disease (HMFD) is available, but it is not known whether the EV71 vaccine cross-protects against Coxsackievirus (CV) infection. Furthermore, although an inactivated circulating CVA16 Changchun 024 (CC024) strain vaccine candidate is effective in newborn mice, the CC024 strain causes severe lesions in muscle and lung tissues. Therefore, an effective CV vaccine with improved pathogenic safety is needed. The aim of this study was to evaluate the in vivo safety and in vitro replication capability of a noncirculating CVA16 SHZH05 strain. The replication capacity of circulating CVA16 strains CC024, CC045, CC090 and CC163 and the noncirculating SHZH05 strain was evaluated by cytopathic effect in different cell lines. The replication capacity and pathogenicity of the CC024 and SHZH05 strains were also evaluated in a neonatal mouse model. Histopathological and viral load analyses demonstrated that the $\mathrm{SHZH05}$ strain had an in vitro replication capacity comparable to the four CC strains. The CC024, but not the SHZH05 strain, became distributed in a variety of tissues and caused severe lesions and mortality in neonatal mice. The differences in replication capacity and in vivo pathogenicity of the $\mathrm{CCO} 24$ and $\mathrm{SHZH05}$ strains may result from differences in the nucleotide and amino acid sequences of viral functional polyproteins P1, P2 and P3. Our findings suggest that the noncirculating SHZH05 strain may be a safer CV vaccine candidate than the CCO24 strain.
\end{abstract}

Key words: Coxsackievirus A16; Pathogenicity; Circulating strain; Non-circulating strain

\section{Introduction}

Hand, foot, and mouth disease (HFMD) mainly affects infants and children, and occasionally occurs in adults worldwide. Several major outbreaks have occurred in Southeast Asia in recent decades (1). Coxsackievirus A16 (CVA16) and enterovirus 71 (EV71), members of the genus Enterovirus and family Picornaviridae, have been identified as the first and second most frequent causes of HFMD, respectively (2). CVA16 infections can lead to severe complications, such as aseptic meningitis, encephalitis, lethal myocarditis, and pneumonia (3-6). Coinfection with CVA16 and EV71 increases genetic recombination between the two viruses, making control of HFMD epidemics even more complex and difficult (7). Such a coinfection may have been responsible for an HFMD outbreak in Fuyang, Anhui Province, China in 2008 (1).

Currently, no antiviral treatment is available for HFMD infection; however, a recently developed EV71 vaccine was consistently immunogenic and provided protection against mild-to-severe disease in a phase III trial (8). An effective vaccine against HFMD should protect against both EV71 and CV infection, but it is not known whether the EV71 vaccine is cross-protective. Maternal vaccination with an inactivated CVA16 Changchun 024 (CC024) strain (a circulating strain that is epidemic in the Changchun city region of China) protected neonatal mice from a series of CVA16 strain challenges. However, the CC024 strain caused severe lesions in the muscle and lung tissues of the newborn mice (9). Therefore, a CV candidate vaccine that protects against HFMD infection and is both effective and pathogenically safe is needed.

In 2010, multiple circulating CVA16 CC strains were isolated from hospitalized patients with HFMD in Jilin Province of Changchun $(9,10)$. A CVA16 SHZH05 strain was also isolated from the city of Shenzhen in Guangdong

Correspondence: Wenyan Zhang: <zhangwenyan@jlu.edu.cn>; Xiao-Fang Yu: <xyu2@jhu.edu>.

${ }^{*}$ These authors contributed equally to this study.

Received August 9, 2014. Accepted December 10, 2014. First published online March 27, 2015. 
Province (11). The SHZH05 strain is considered to be a noncirculating CVA16 strain because it shares the same recombination pattern and forms a close cluster with the CVA16 circulating CC strains, but is not closely related to the prototype CVA16 G10 (10,12). It is not known why infection with only some CVA16 strains results in neurological complications and even death. It is also unclear whether the CVA16 circulating and noncirculating strains differ in replication capacity and pathogenesis. In this study, we aimed to determine whether the noncirculating $\mathrm{SHZHO5}$ strain is safe for newborn mice while maintaining a replication capacity similar to that of the circulating $\mathrm{CC}$ strains in different cell lines.

\section{Material and Methods}

\section{Ethics statement}

Approval for this study was obtained from the Ethics Committee at the First Hospital of Jilin University. Written informed consent was obtained from the parents of all the children involved in the study. All animal protocols were approved by the Ethics Committee of Jilin University Institute of Animal Care and Use. All specimens were confirmed to be positive for the CVA16 VP1 conserved region by a Coxsackievirus $\mathrm{A} 16$ polymerase chain reaction (PCR) kit (DAAN Gene Co., Ltd. of Sun YatSen University, China) (13).

\section{Cells and viruses}

The C6 cell line was a gift from the Tumor Center of First Hospital of Jilin University, Chanagchun, China. Vero, baby hamster kidney (BHK), C6 and L929 cell lines were purchased from the American Type Culture Collection (ATCC, USA) and cultured in Dulbecco's modified Eagle's medium (DMEM; Gibco, Invitrogen, USA) supplemented with $10 \%$ fetal bovine serum (FBS; Gibco, Invitrogen) at $37^{\circ} \mathrm{C}$ in an atmosphere containing $5 \% \mathrm{CO}_{2}$. The CVA16 strains CC024, CC045, CC090 and CC163 that were isolated in 2010 from throat swabs of hospitalized HFMD patients in Changchun were propagated in a Vero cell line. Viral samples were diluted in DMEM and passed through a $0.22-\mu \mathrm{m}$ filter before infection of Vero cells. Viruses were harvested and continuously passaged until a cytopathic effect (CPE) was observed. The SHZH05 strain (GenBank accession No. EU262658.1) was a gift from Professor Qi Jin of the Institute of Pathogen Biology, Chinese Academy of Medical Sciences and Peking Union Medical College, Beijing, China.

\section{Isolation and primary neonatal mouse lung cell culture}

Lung tissue of 1-day-old specific pathogen-free (SPF) neonatal ICR mice from the Experimental Animal Center, College of Basic Medicine, Jilin University, was minced and kept overnight in $2 \mathrm{~mL}$ of $0.25 \%$ trypsin-EDTA at $4{ }^{\circ} \mathrm{C}$. The suspension was diluted in $2 \mathrm{~mL}$ of DMEM with $15 \%$
FBS and filtered through several layers of gauze to remove tissue pieces. The suspension was then centrifuged at $200 \mathrm{~g}$ for $5 \mathrm{~min}$ to collect the cells, which were then resuspended in DMEM with $15 \%$ FBS in a cell culture flask at $37^{\circ} \mathrm{C}$ in a humidified atmosphere containing $5 \% \mathrm{CO}_{2}$. The cells obtained from this primary culture were named $\mathrm{ML}-1$.

\section{Virus titration}

Virus titers were expressed as the median tissue culture infectious dose $\left(T_{C} I_{50}\right)$ obtained by end-point dilution. Serially diluted viruses were added to Vero cells grown in 96-well plates $(n=8)$ that were incubated for 7 days at $35^{\circ} \mathrm{C}$. The $\mathrm{TCID}_{50}$ values were measured by determining the CPE in infected Vero cells and calculated by the Reed-Muench method.

\section{In vitro infection}

Monolayers of Vero, BHK, C6, L929, and ML-1 cells cultured in 24-well plates were infected with the $\mathrm{SHZH05}$, CC024, CC045, CC090 or CC163 strains or mockinfected with DMEM media. The infected cells were maintained at $37^{\circ} \mathrm{C}$ in a $5 \% \mathrm{CO}_{2}$ atmosphere for $96 \mathrm{~h}$.

\section{Neonatal mouse infection}

One-day-old SPF ICR neonatal mice weighing 1.8$2.0 \mathrm{~g}$ were randomly allocated to 3 groups of $8-10$ mice from single litters for infection with: a) $10^{5.5} \mathrm{CCID}_{50} / \mathrm{mL}$ CC024 strain, b) $10^{5.5} \mathrm{CCID}_{50} / \mathrm{mL}$ SHZH05 strain, or c) mock infection with DMEM. The neonatal mice were intracerebrally inoculated with $20 \mu \mathrm{L}$ of 10 -fold serially diluted virus or mock inoculated with DMEM. The severity of clinical disease was scored as: 0 , healthy; 1 , lethargy and inactivity; 2, wasting; 3, limb-shaking weakness; 4, hind limb paralysis; 5, moribund or dead. Body weight, activity, occurrence of limb paralysis, morbidity, and death were monitored and recorded until 21 days post-infection. The median lethal dose $\left(L D_{50}\right)$ was calculated by the Reed-Muench method.

\section{Histopathological analysis}

At 21 days post-infection, after disease severity scoring, samples of brain, lung, spinal and hind-limb muscle, liver, kidney, spleen, heart, and intestine tissue were examined. Tissue was obtained from a) 3 dead mice infected with $10^{5.5} \mathrm{CCID}_{50} / \mathrm{mL}$ CC024 strain, b) 3 mice infected with $10^{5.5} \mathrm{CCID}_{50} / \mathrm{mL} \mathrm{SHZHO5}$ strain, and c) three mock-infected mice. Tissue samples were fixed in $10 \%$ formalin for 3-5 days, dehydrated through an ethanol gradient, embedded in paraffin, sectioned at $4 \mu \mathrm{m}$, and stained with hematoxylin and eosin. Histopathological analysis was performed by light microscopy (CKX-31, Olympus, Japan).

\section{Viral loads in infected neonatal mouse tissue}

Heart, liver, spleen, lung, kidney, brain, intestine, 


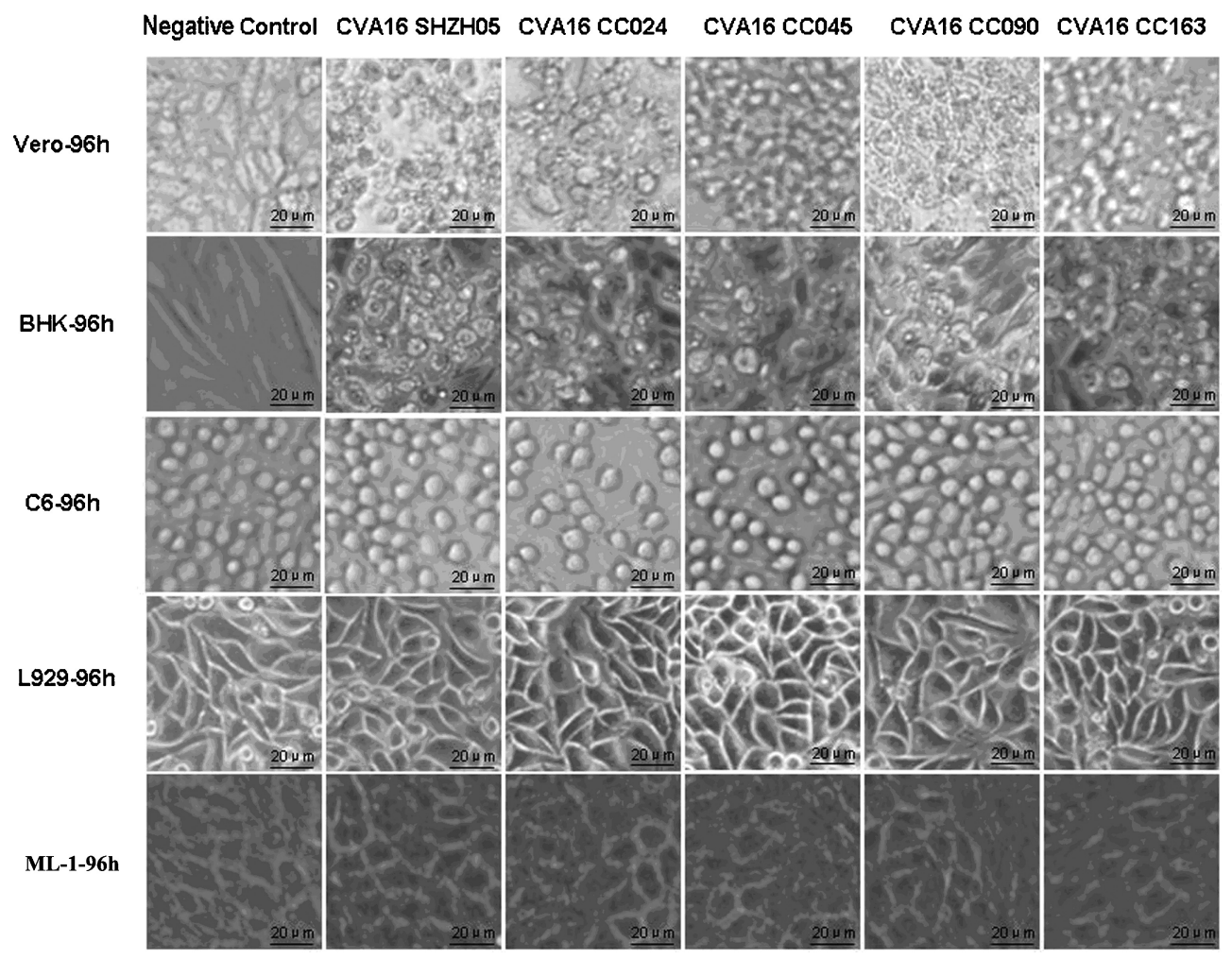

Figure 1. Coxsackievirus A16 (CVA16) replication in vitro. Vero, BHK, C6, L929, and neonatal mouse lung ML-1 cells were infected with the circulating CVA16 Changchun (CC) CC024, CC045, CC090, or CC163 strains or the noncirculating CVA16 SHZH05 strain for $96 \mathrm{~h}$. SHZH05 and all four CC strains caused a cytopathic effect (CPE) in BHK and Vero cells, but not the other three cell lines.

spinal and hind-limb muscle tissue and blood were collected from 3 mice in each group on days 2, 3, 4 and 5 post-infection. The tissue samples were weighed, homogenized in sterile phosphate buffered saline, disrupted by freeze-thawing, and centrifuged at $7500 \mathrm{~g}$ for $10 \mathrm{~min}$. All samples were treated with TRIZOL (Invitrogen) for RNA extraction, and the viral load was determined by quantitative real-time polymerase chain reaction (qRT-PCR) and expressed as $\log _{10}$ copies $/ \mathrm{mg}$ tissue or $\log _{10}$ copies $/ \mathrm{mL}$ blood.

\section{RNA extraction and qRT-PCR}

For qRT-PCR, viral RNA was extracted from fresh tissue homogenates using TRIZOL (Invitrogen), and cDNA was generated using a high-capacity cDNA reverse transcription kit (Applied Biosystems, Inc., USA) and oligo$\mathrm{d}(\mathrm{T})_{18}$ primers according to the supplier's instructions. Primers designed according to the VP1 conserved region sequences of CVA16 were: CVA16-F1, CATGCAGCGC TTGTGCTT; CVA16-F2, CATGCAACGACTGTGCTTTC; CVA16-R1, CACACAATTCCCCCGTCTTAC; and CVA16R2, CATAATTCGCCCGTTTTGCT. The SYBR Greenbased qRT-PCR was carried out on a Mx3005P machine
(Agilent Technologies Stratagene, USA) using the doublestrand DNA-binding dye method with SYBR Green PCR Master Mix (Applied Biosystems, Inc.). Each $20 \mu \mathrm{L}$ reaction mixture contained $10 \mu \mathrm{L}$ of SYBR Premix, $0.2 \mu \mathrm{L}$ each of $\mathrm{F} 1, \mathrm{R} 1, \mathrm{~F} 2$, and R2 (all $10 \mu \mathrm{M}$ ), $7.2 \mu \mathrm{L}$ of double-distilled $\mathrm{H}_{2} \mathrm{O}$, and $2 \mu \mathrm{L}$ of cDNA template. Cycling conditions were: $50^{\circ} \mathrm{C}$ for $2 \mathrm{~min}$, then $95^{\circ} \mathrm{C}$ for $10 \mathrm{~min}$, followed by 50 cycles of $95^{\circ} \mathrm{C}$ for $15 \mathrm{~s}$ and $60^{\circ} \mathrm{C}$ for $1 \mathrm{~min}$. The melting curve analysis was performed at $90^{\circ} \mathrm{C}$ for $1 \mathrm{~min}$, then $55^{\circ} \mathrm{C}$ for $30 \mathrm{~s}$, and $95^{\circ} \mathrm{C}$ for $30 \mathrm{~s}$.

The copy number of the target cDNA in the qRT-PCR was determined by a standard curve of 10 -fold serially diluted nonlinearized plasmid DNAs containing the target VP1 sequence (ranging from 102 to 109 copies). Absolute RNA copy numbers were calculated by standard dilution curves of the plasmids containing the target sequence. The sensitivity of the assay (i.e., limit of detection) was determined as the lowest copy number that was amplified consistently within the linear portion of the standard curve.

\section{Gene sequence alignment}

The nucleotide and amino acid sequences of the 


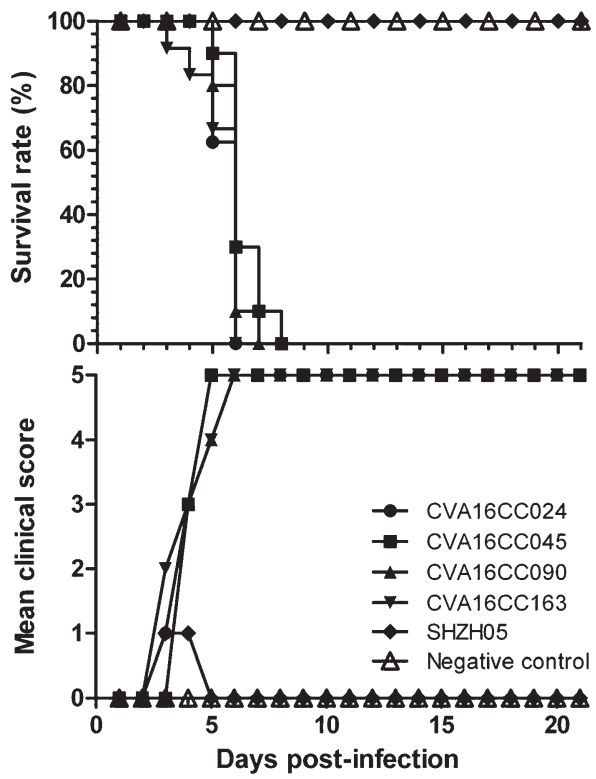

Figure 2. Clinical scores and survival of neonatal mice infected with circulating Coxsackievirus A16 (CVA16) Changchun (CC) strains CC024, CC045, CC090, or CC163, or the noncirculating CVA16 SHZH05 strain. Neonatal mice $(n=8-10$ per litter) were intracerebrally infected with $20 \mu \mathrm{L}$ of $10^{5.5} \mathrm{CCID}_{50} / \mathrm{mL}$ of each of the CVA16 virus strains, or mock infected with DMEM. The mean clinical scores and survival rates were monitored and recorded daily for 21 days postinfection.

circulating $\mathrm{CCO} 24$ and noncirculating $\mathrm{SHZH05}$ strains were aligned using the DNAMAN software (Version 6.0, USA).

\section{Statistical analysis}

Data for the viral loads and clinical scores were compared using a nonparametric one-way analysis of variance (ANOVA). Survival rates were evaluated by a log-rank test. Results are reported as means \pm SE. $\mathrm{P}<0.05$ was considered to be statistically significant.

\section{Results}

\section{Circulating $\mathrm{CC}$ and noncirculating $\mathrm{SHZH05}$ strain replication in vitro}

Vero, BHK, C6, L929, and ML-1 cells were infected with one of the four CC strains or with the $\mathrm{SHZH05}$ strain at $10^{5.5} \mathrm{CCID}_{50} / \mathrm{mL}$. The results demonstrated that the SHZH05 strain and the CC024, CC045, CC090, and CC163 strains induced similar CPEs in Vero and BHK cells at $96 \mathrm{~h}$ post-infection (Figure 1). However, no CPE was observed in C6, L929 and ML-1 cells infected by any of the virus strains (Figure 1). The primary mouse ML-1 cells were used as a control to rule out possible resistance to CVA16 infection by immortalized cells and to test the sensitivity of the ML-1 cells to CVA16.

\section{Circulating CC strains, but not the noncirculating SHZH05 strain, induced lethal symptoms and mortality in neonatal mice}

To test whether the circulating $(\mathrm{CCO} 24)$ and noncirculating ( $\mathrm{SHZH05)}$ strains differed in virulence, the disease scores and the mortality rates of newborn mice were recorded until 21 days post-infection. All mice infected with the CC024, CC045, CC090 or CC163 strain became sick by day 3 post-infection, had a mean clinical score of grade 1 , and mortality reached $100 \%$ by day 6 (Figure 2). As expected, the mock-infected mice had clinical scores of grade 0 and a $100 \%$ survival rate.

The mice challenged with $20 \mu \mathrm{L}$ of the CC024 strain at $10^{5.5} \mathrm{CCID}_{50} / \mathrm{mL}$ showed signs of illness on days 3 to 10
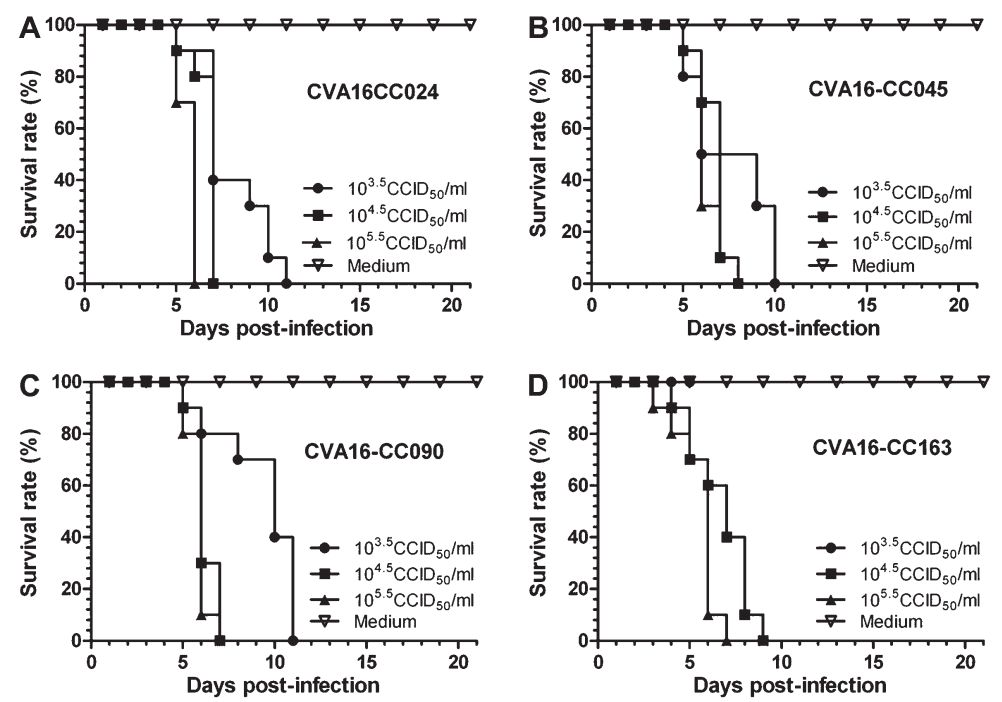

Figure 3. Survival of neonatal mice infected with circulating Coxsackievirus A16 (CVA16) Changchun (CC) strains CC024, CC045, CC090 or CC163 at different doses. Neonatal mice in each group ( $n=8-10$ per litter) were intracerebrally infected with each of the above CVA16 strains at different doses from $10^{3.5}$ to $10^{5.5} \mathrm{CCID}_{50} / \mathrm{mL}$, or mock infected with DMEM medium. 


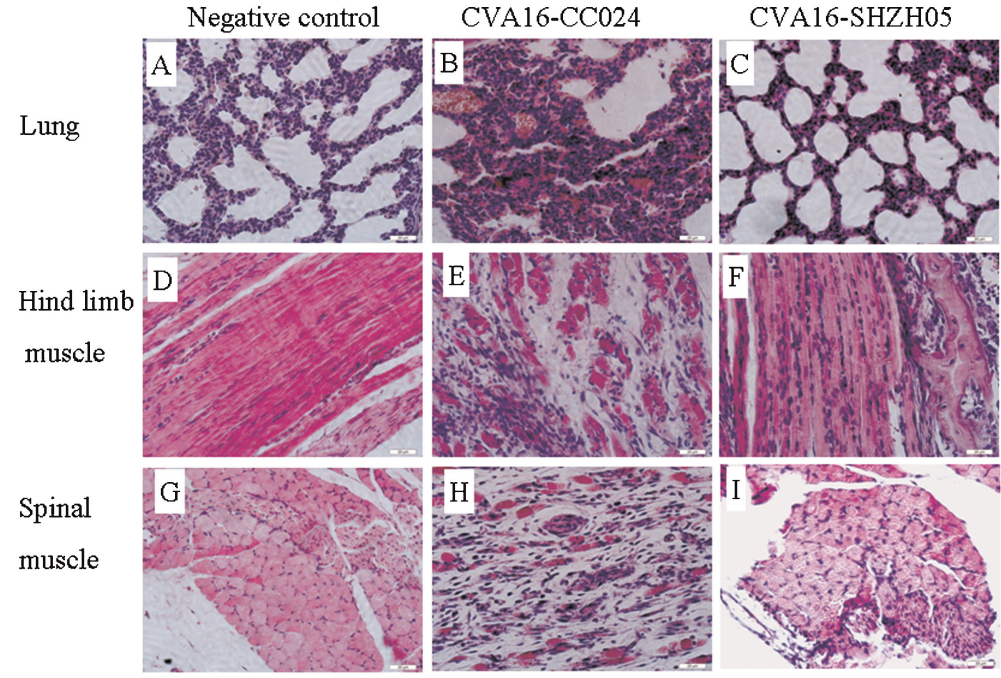

Figure 4. Histological analysis of tissues from Coxsackievirus A16 (CVA16)-infected neonatal mice. One-day-old SPF ICR mice were infected intracerebrally with $20 \mu \mathrm{L}$ of $10^{5.5} \mathrm{CCID}_{50} / \mathrm{mL}$ of the $\mathrm{CCO24}$ or the SHZH05 strain, or mock infected with DMEM. No histological change was observed in the lung tissue, hind limb muscle, and spinal skeletal muscle of the $\mathrm{SHZHO5}$ strain-infected $(C, F$, and $I)$ or mock-infected mice $(A, D$, and $G)$. Mice with grade 5 clinical symptoms infected with the $\mathrm{CCO} 24$ strain virus exhibited severe alveolar shrinkage and vascular congestion (arrow) in the lung tissue $(B)$, severe necrosis and loose muscle fibers in the hind limb muscle $(E)$ and spinal skeletal muscle $(H)$. Magnification $400 \times$. All experiments were repeated three times.

(clinical scores of grade 1 to 5 ) and $100 \%$ mortality on day 7 (Figures 2 and 3A). The circulating CC024 strain demonstrated dose-dependent disease symptoms and mortality; dose-dependent mortality was also observed with the other circulating CC strains (CC045, CC090, and CC163). Mice infected with $10^{3.5} \mathrm{CCID}_{50} / \mathrm{mL}$ strains CC163, CC045, or CC090 started to die on days 4 or 5 , with $100 \%$ mortality on days 9,10 , and 11 , respectively (Figure 3B,C,D). Mice infected with circulating CC strains at $10^{4.5} \mathrm{CCID}_{50} / \mathrm{mL}$ or $10^{5.5} \mathrm{CCID}_{50} / \mathrm{mL}$ exhibited increasing morbidity on days 3 to 5 , with $100 \%$ mortality on days 7 to 8 (Figure 3B,C,D). All the CC strain infections were lethal, but $\mathrm{SHZHO5}$ strain infections failed to induce illness or death in the neonatal mice (data not shown).

\section{Pathological analysis of the SHZH05 and CC024} strain infections in neonatal mice

Pathological analysis was carried out in brain, lung, spinal and hind-limb muscle, liver, kidney, spleen, heart and intestine tissues from mice infected with the CC024 strain, which was considered representative of the four CC strains, and the SHZH05 strain. CC024 infection caused obvious lung tissue lesions, including severe alveolar shrinkage (Figure 4B), scattered areas of pulmonary fibrosis (Figure 4B), pulmonary edema, vascular dilation and congestion, severe necrosis of skeletal muscle, muscle bundle fracture, and dissolution of muscle fibers (Figure 4E and $\mathrm{H}$ ). No pathological changes were observed in tissues of mice infected with the SHZHO5
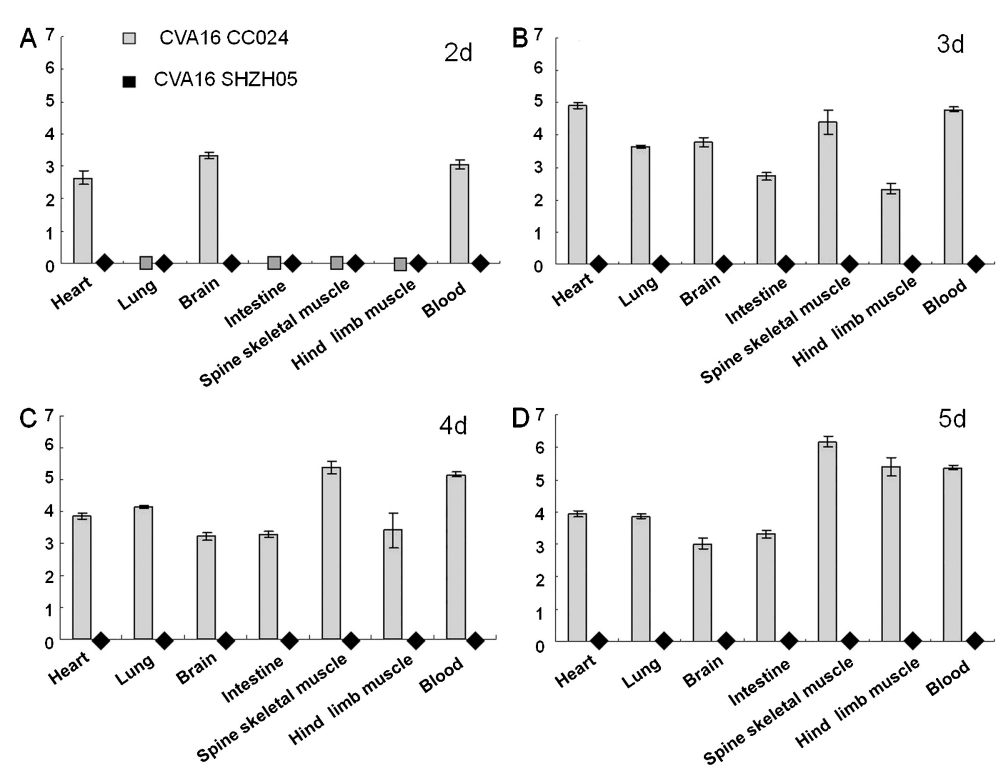

Figure 5. Mean viral loads in tissues of Coxsackievirus A16 (CVA16) CC024 or CVA16 SHZH05 strain-infected neonatal mice with $20 \mu \mathrm{L}$ of $10^{5.5} \mathrm{CCID}_{50} / \mathrm{mL}$ at $2,3,4$, or 5 days (d) postinfection. Viral loads were assessed by qRT-PCR amplification of the viruses in the heart, lung, brain, intestine, spine skeletal muscle, hind limb muscle tissues and the blood from the infected mice. Results are reported as $\log _{10}$ copies/mg tissue or $\log _{10}$ copies $/ \mathrm{mL}$ blood \pm SD. There were 3 mice per group; experiments were repeated 3 times. 
Table 1. Genetic differences between CVA16 CC024 and SHZH05 strains.

\begin{tabular}{|c|c|c|}
\hline \multirow[t]{2}{*}{ Gene segments } & \multicolumn{2}{|c|}{ Differences } \\
\hline & Nucleotides & Amino acids \\
\hline 5'UTR & $\begin{array}{l}\text { 3.76\%: G26A, T29C, G67A, A89G, C96T, C97T, } \\
\text { T100C, C104T, T120C, G122A, T123C, A149G, } \\
\text { T158C, T169C, C199T, G226A, C241T, T271C, } \\
\text { C332T, C424T, C448T, G491A, C579T, T580C, } \\
\text { T584C, A660G, G675A, G742A }\end{array}$ & \\
\hline P1 & $6.54 \%$ & $\begin{array}{c}0.7 \% \text {, changes are: R51K, K52R, T295A, H364R, } \\
\text { N464S, R850K }\end{array}$ \\
\hline P2 & $6.34 \%$ & $\begin{array}{c}1.21 \% \text {, changes are: S37T, M165V, S180N, } \\
\text { K191R, V345T, K355R, R529K }\end{array}$ \\
\hline P3 & $7.04 \%$ & $\begin{array}{c}\text { 1.73\%, changes are: N11S, S48P, S299P, } \\
\text { A320V, Y322H, S328N, T335S, I383T, Y397H, } \\
\text { F434L, R570K, I595V, R637K }\end{array}$ \\
\hline
\end{tabular}

strain (Figure $4 \mathrm{C}, \mathrm{F}, \mathrm{I}$ ) or of mock infected neonatal mice (Figure 4A,D,G). These results demonstrate that the CC024 strain had a strong tropism for muscle and lung tissues and was responsible for the severe lesions in these tissues, but that the $\mathrm{SHZHO5}$ strain did not have a muscle tropism or cause any lesions in this neonatal mouse model.

\section{Kinetics of viral replication in various tissues of the SHZH05 or CC024 strain-infected neonatal mice}

To further understand the replication and distribution of the $\mathrm{SHZHO}$ and $\mathrm{CCO} 24$ strains in infected mice, we determined the viral loads in various tissues on different days postinfection. At 2 days postinfection, viral loads were detected only in the heart $\left(10^{2.652}\right.$ copies $\left./ \mathrm{mg}\right)$, brain $\left(10^{3.322}\right.$ copies $\left./ \mathrm{mg}\right)$ and blood $\left(10^{3.052} \mathrm{copies} / \mathrm{mL}\right)$ of CC024 strain-infected mice (Figure 5 ). The viral loads were increased in the heart, lung, brain, intestine, spinal and hind-limb muscle, and blood of the CC024 straininfected mice at 3 and 4 days postinfection (Figure 5). However, no viruses were detected in any tissues of SHZH05 strain-infected or mock-infected mice.

\section{Genetic sequence alignment}

Gene sequence analysis by DNAMAN software revealed a $3.76 \%$ diversity between the $\mathrm{SHZHO5}$ and CC024 strains in the $5^{\prime}$ untranslated regions (UTR). Approximately $6.54,6.34$, and $7.04 \%$ diversity was seen in the viral genome nucleotide sequences of viral functional polyproteins $\mathrm{P} 1, \mathrm{P} 2$, and $\mathrm{P} 3$, respectively. Diversities of $0.7,1.21$ and $1.73 \%$ were observed in the corresponding P1, P2, and P3 amino acid sequences (P1: R51K, K52R, T295A, H364R, N464S, R850K; P2: S37T, M165V, S180N, K191R, V345T, K355R, R529K; P3: N11S, S48P, S299P, A320V, Y322H, S328N, T335S, I383T, Y397H, F434L, R570K, I595V, R637K), respectively (Table 1 ).

\section{Discussion}

An enterovirus 17 (EV71) vaccine has been developed (8), but whether this vaccine candidate cross-protects against $\mathrm{CV}$ infection remains unknown. Furthermore, a $\mathrm{CV}$ vaccine candidate developed in a mouse model was found to cause severe lesions in muscle and lung tissue (9). We evaluated in vitro viral replication in different cell lines and pathogenicity in a neonatal mouse model to determine the pathogenic safety and replication capacity of the noncirculating CVA16 $\mathrm{SHZH05}$ and circulating CVA16 Changchun (CC) strains. We found that the $\mathrm{SHZH05}$ strain had a replication capability similar to the four $\mathrm{CC}$ strains in vitro. However, the $\mathrm{SHZH05}$ strain was less pathogenic than the $\mathrm{CC}$ strains in the neonatal mouse model. All four CC strains, but not the $\mathrm{SHZH05}$ strain, caused tissue-specific pathological changes and lethal infections.

We found that the SHZH05 strain had similar replication capacities in each of the cell lines, including the ML-1 cell primary cultures. However, histopathological analysis and viral load measurements confirmed that it failed to replicate in neonatal mouse tissues. The capacity to replicate in vitro and the improved safety indicate that the $\mathrm{SHZHO} 5$ strain may be a better $\mathrm{CV}$ candidate than the CC024 strain vaccine (9). It is still not clear why the circulating CC024, but not the noncirculating SHZH05 virus strain, caused death in the neonatal mice. One reason might be that some genetic changes have occurred within the $\mathrm{SHZHO5} \mathrm{genome,} \mathrm{resulting} \mathrm{in} \mathrm{a} \mathrm{failure}$ of the receptors on the surface of neonatal mouse cells to recognize the SHZH05 strain. In fact, the CVA16 and EV71 strains are unable to infect some mouse-derived cell lines, such as L929 and Ltr246, because there are no CVA16- or EV71-related receptors on the cell surface (14). It should be noted that comparative analysis of the SHZH05 and CVA16 CC024 strains revealed differences 
in the nucleotide and amino acid sequences of functional polyproteins P1, P2 and P3 (Table 1). These changes might account for the difference in pathogenicity of the SHZH05 and CC024 strains. Further study of the lack of infectivity of the $\mathrm{SHZHO5}$ strain in neonatal mice is important for the development of a $\mathrm{CV}$ vaccine.

The pathological analysis of infected neonatal mouse tissues indicated a CC024 strain tropism toward lung and muscle, which is in line with the findings of a previous study (9). Furthermore, viral load analysis demonstrated that the CC024 strain distributed among all of the tissues examined, but that the SHZHO5 strain was not found in any of the tissues. These results further indicate that the noncirculating $\mathrm{SHZH05}$ strain might be a safer $\mathrm{CV}$ vaccine candidate, the efficacy of which would be worth testing in a further study.

The noncirculating CVA16 SHZH05 strain had a replication capacity similar to the four circulating CVA16 CC strains in vitro, but unlike the circulating CC024 strain, it failed to replicate or cause adverse pathological changes and mortality in vivo. These differences might

\section{References}

1. Mao $Q$, Wang $Y$, Yao $X$, Bian $L$, Wu $X, X u M$, et al. Coxsackievirus A16: Epidemiology, diagnosis, and vaccine. Hum Vaccin Immunother 2014; 10: 360-367, doi: 10.4161/ hv.27087.

2. Repass GL, Palmer WC, Stancampiano FF. Hand, foot, and mouth disease: Identifying and managing an acute viral syndrome. Cleve Clin J Med 2014; 81: 537-543, doi: 10.3949/ccjm.81a.13132.

3. Goto K, Sanefuji M, Kusuhara K, Nishimura Y, Shimizu H, Kira R, et al. Rhombencephalitis and coxsackievirus A16. Emerg Infect Dis 2009; 15: 1689-1691, doi: 10.3201/ eid1510.090594.

4. Wang CY, Li LF, Wu MH, Lee CY, Huang LM. Fatal coxsackievirus A16 infection. Pediatr Infect Dis J 2004; 23: 275-276, doi: 10.1097/01.inf.0000115950.63906.78.

5. Legay F, Leveque N, Gacouin A, Tattevin P, Bouet J, Thomas $R$, et al. Fatal coxsackievirus A-16 pneumonitis in adult. Emerg Infect Dis 2007; 13: 1084-1086, doi: 10.3201/ eid1307.070295.

6. Eyckmans $T$, Wollants $E$, Janssens $A$, Schoemans $H$, Lagrou K, Wauters J, et al. Coxsackievirus A16 Encephalitis during Obinutuzumab Therapy, Belgium, 2013. Emerg Infect Dis 2014; 20: 913-915, doi: 10.3201/eid2005.131766.

7. Wang X, Zhu C, Bao W, Zhao K, Niu J, Yu XF, et al. Characterization of full-length enterovirus 71 strains from severe and mild disease patients in northeastern China. PLOS One 2012; 7: e32405, doi: 10.1371/journal.pone.0032405.

8. Zhu FC, Xu WB, Xia JL, Liang ZL, Liu Y, Zhang XF, et al. result from differences in nucleotide and amino acid sequences of the functional $\mathrm{P} 1, \mathrm{P} 2$ and $\mathrm{P} 3$ polyproteins in the two strains. Our findings demonstrated that the CVA16 SHZH05 strain may be a potentially safer CV vaccine candidate for prevention of HMFD than other available strains.

\section{Acknowledgments}

The authors thank Professor Qi Jin from the Institute of Pathogen Biology, Chinese Academy of Medical Sciences for the CVA16 SHZH05 strain. The research was supported in part by the National Natural Science Foundation of China (No. 31270202), the Chinese Ministry of Science and Technology (No. 2012CB911102 and No. 2013ZX0001-005), the Health and Family Planning Commission of Jilin Province (No. 2013Z066), the Chinese Ministry of Education (No. IRT1016) and the Key Laboratory of Molecular Virology of Jilin Province (No. 20102209).
Protection from lethal challenge in a neonatal mouse model by circulating recombinant form coxsackievirus $\mathrm{A} 16$ vaccine candidates. J Gen Virol 2014; 95: 1083-1093, doi: 10.1099/ vir.0.065003-0.

9. Li J, Chang J, Liu X, Yang J, Guo H, Wei W, et al. Protection from lethal challenge in a neonatal mouse model by circulating recombinant form coxsackievirus A16 vaccine candidates. J Gen Virol 2014; 95: 1083-1093, doi: 10.1099/ vir.0.063560-0.

10. Wei W, Guo H, Li J, Ren S, Wei Z, Bao W, et al. Circulating HFMD-associated coxsackievirus A16 is genetically and phenotypically distinct from the prototype CV-A16. PLoS One 2014; 9: e94746, doi: 10.1371/journal.pone.0094746.

11. Wu Z, Yang F, Zhao R, Zhao L, Guo D, Jin Q. Identification of small interfering RNAs which inhibit the replication of several Enterovirus 71 strains in China. J Virol Methods 2009; 159: 233-238, doi: 10.1016/j.jviromet.2009.04.002.

12. Zhao $K$, Han $X$, Wang $G$, Hu W, Zhang $W$, Yu XF. Circulating coxsackievirus A16 identified as recombinant type A human enterovirus, China. Emerg Infect Dis 2011; 17: $1537-1540$.

13. Hagiwara A, Tagaya I, Yoneyama T. Epidemic of hand, foot and mouth disease associated with enterovirus 71 infection. Intervirology 1978; 9: 60-63, doi: 10.1159/000148922.

14. Yamayoshi S, Koike S. Identification of a human SCARB2 region that is important for enterovirus 71 binding and infection. J Virol 2011; 85: 4937-4946, doi: 10.1128/ JVI.02358-10. 